be very complete. We are of opinion, however, that the bullet took a downward and backward course after its entry and passed below the diaphragm, after which it struck the spinal column, and passed through the diaphragm on the right side. The symptoms from which the patient suffered while at the C.C.S. might be interpreted as being due to injury to the liver and haemothorax, and although no positive evidence as to the former was found at necropsy, the pleural adhesions present on the right side might be attributable to spontaneous cure of the latter condition. The various findings of the Boards certainly suggest diaphragmatic injury, and with regard to the $x$-ray appearances the site of the bullet between the eleventh and twelfth dorsal vertebrae, with possible injury of the eleventh dorsal vertebra, affords corroborative evidence of our suggestion as to the path of the bullet. Moreover, since the aneurysmal sac extended between the origin of the superior mesenteric artery and the under surface of the diaphragm on the right side, its site corresponds to the lower half of the twelfth and the upper half of the first lumbar vertebra. This point is sufficiently near the final position of the bullet (in the right lung base immediately above the diaphragm) to indicate an association between the path of the bullet and the aneurysm. As to the exact nature of the injury which the aortic wall must have sustained, one is left in considerable doubt, and it is remarkable that in the circumstances damage to either aorta or inferior vena cava did not result in rapid death. Since this did not happen, the formation of an aneurysm may have been due to: (1) grazing injury to the aortic wall such as might be produced by $(a)$ the passage of a bullet, (b) a dislodged spicule of bone ; or (2) inflammatory reaction in the track of the bullet. The combination of cicatricial weakening from the injury and the intra-aortic pressure would necessarily lead to a progressive local dilatation of the aorta.

We sent the bullet to Professor Sydney Smith of the Forensic Medicine Department of Edinburgh University, to whom we are indebted for the following report:

"It is a Danish bullet composed of a lead core with steel and nickelled casing. It was fired from a German rifle. It was not a direct hit but was a ricochet, having struck a hard surface before it hit the victim. This would give it a more eccentric course and would explain its direction in the body. After entering in the region of the seventh rib to the left of the middle line, it no doubt struck the aorta a glancing blow, which caused a certain amount of bruising; then passed between the pillars of the diaphragm into the chest cavity. The damage to the wall of the aorta would lead to fibrosis and stretching."

We are unable to account for the clinical latency of the condition with regard to dislodgement of portions of thrombus from the sac, which might have occurred at any time and ultimately caused death. The condition of calculous hydronephrosis, which was present, was apparently an entirely separate pathological process, and can be associated neither with the bullet wound nor with the final illness.

\section{OBSERVATIONS ON THE TREATMENT OF TETANUS}

\section{(WITH SPECIAL REFERENCE TO TETANUS ANTITOXIN)*} BY

\section{B. B. YODH, M.B., M.R.C.P., D.T.M. AND H. HONORARY PHYSICIAN, J. J. HOSPITAL, BOMBAY}

My purpose in dealing with this subject is not to describe any new method of treatment or to claim any startling results, but to take stock of the present situation regarding the various methods of treatment advocated and of the methods tried in this group of cases. A continuous charge of the male tetanus wards for five years has enabled me to observe a fairly large number of cases, and to vary, and perhaps improve, the technique of treatment from time to time.

\section{Methods of General Treatment}

The general treatment is very important, and I propose to deal with this first.

The fatal effects of tetanus are principally due to: (1) poisoning of the vital centres, causing respiratory and cardiac failure; (2) exhaustion due to continuous muscular spasm and repeated seizures on the top of it, the latter being intensely aggravated by peripheral stimuli, even very light ones. To minimize this second factor requires very careful, painstaking, and intelligent nursing.

A well-ventilated dark room is very important, as by this means the external stimuli are considerably reduced. In the construction of small tetanus wards the walls should be painted dark, window shutters obliquely placed, and an exhaust fan provided. There should be silence in the ward. The slightest noises, such as caused by shuffling of the feet or jingling of glass, bring on spasms. Bromides, chloretone, chloral hydrate, hyoscyamus, etc., are the usual sedatives employed. Large doses are required at the beginning. Morphine with atropine may be useful when the spasms are very severe, and during the early stages.

* Being the Lord Reay Lectures delivered at the Grant Medical College in November, 1931.
Too much morphine, however, should be avoided, especially when pulmonary complications are present. 'The prolonged use of these sedatives is attended with some danger, and one has come across several cases of drowsiness and even coma after the spasms.have passed awayprobably as a cumulative effect of the sedatives.

\section{、 Chloroform and Special Drugs}

Although all the textbooks prescribe it, and in some French journals a few cases have been reported in which enormous doses have been used with apparently good results, I do not advocate the use of chloroform for the relief of spasms. Our own experience of chloroform, which was used in earlier cases, is not satisfactory, as pulmonary oedema supervened in a fair number of these cases with fatal results. It is, of course, difficult to prove that chloroform was the factor concerned, but such is the clinical impression. We now avoid chloroform even while performing cistern or lumbar puncture. Anaesthesia, which may be required in some cases for the extreme rigidity and frequent spasms, can easily be obtained by the use of liq. somniferus (Stella and Co.). This is quite safe and gives immediate unconsciousness which acts for a short time only. It has been used in about a dozen instances in this series, and no ill effects have been observed.

Injection of special drugs, such as carbolic acid and magnesium sulphate, has been recommended for the relief of spasms, but they have not been used in this series of cases at all. On looking up the recent literature one does not find any great claims made for them, although the older textbooks recommend them. Recently two articles have been published regarding the carbolic acid treatment (Bacelli) of tetanus. Pilloni ${ }^{11}$ has treated fiftythree cases over a period of twelve years, with 70 per cent. cures, by injection of phenol 0.5 gram for the first two days, followed by smaller doses. He had only three cases of nephritis in the series. Suvansu ${ }^{12}$ has treated fourteen consecutive cases of tetanus with intrathecal injections of carbolic acid, with four deaths, and has not 
included two moribund cases in the series. The dosage has been 30 to $40 \mathrm{c} . \mathrm{cm}$. of 1 in 400 carbolic acid solution in adults, and 12 to $20 \mathrm{c.cm}$. in children. Small doses of serum had been administered in some of these cases, although not intrathecally. This, like Pilloni's, is a very small series of cases.

The Wound, Diet, etc.

Local treatment is important. A dirty, lacerated wound, likely to be contaminated with other bacteria, offers a favourable spot for the growth of the tetanus bacilli, which seem to grow better in symbiosis with aerobes. The pus cocci in a tetanus wound present phagocytosis of tetanus bacilli, and these latter multiply and produce more toxin. Excision of the wound area is a better method than treating it with antiseptics. If it is not possible to excise, a thorough cauterization is essential. Experiments in guinea-pigs tend to show that the subcutaneous injections of oxygen deep into the wound may delay or prevent the onset of tetanus. ${ }^{2}$ Presence of oxygen in the wound would certainly prevent the multiplication of the organisms, and its use in deep wounds therefore is certainly rational, both as a prophylactic and as a curative in the early stages. It has not been used in this series of cases, as the vast majority of them were well advanced.

As regards the excretory functions, retention of urine is rare, so that a catheter seldom has to be passed. Constipation, however, is the rule, and a simple daily enema is required to empty the bowels.

With the ordinary lockjaw only fluid diet is possible, while sometimes with complete lockjaw and oesophageal spasm even that is out of the question for a time. Fluids, such as milk, weak tea, barley water, etc., are given at two-hourly intervals and in small quantities, and, as a rule, they are taken without great difficulty. Nasal feeding may have to be considered, but the passage of a catheter through the nose has, in one of our cases, brought on a fatal spasm, so that one is chary of ordering this measure. Rectal saline alone may have to be relied on for nourishment for twenty-four or forty-eight hours at the most.

A marked increase in the pulse rate and the onset of respiratory failure with pulmonary oedema sometimes yield to the timely use of digitaline and atropine subcutaneously. These should be injected early and in fairly large doses-1/100 grain of the former and 1/50 grain of the latter every three hours.

Before the introduction of tetanus antitoxin the main line of treatment was the use of sedatives in large doses with injections of magnesium sulphate or carbolic acid. The results of this line of treatment have never been satisfactory if a large series of consecutive cases is considered. The mortality rate in a large series has always been above 60 per cent.

\section{Use of Tetanus Antitoxin}

As the results of the treatment adopted before the introduction of the antitoxin were very poor, it was hoped that the use of the antitoxin would revolutionize the treatment of tetanus, as happened in the case of diphtheria. Unfortunately this has not been so, and some authorities go so far as to deny that the antitoxin is of any value. It is useful, therefore, to consider first if the use of the antitoxin is rational.

\section{EXPt:RIMENTAL WORK}

The specificity of the antitoxin was first experimentally proved by Kitasato, discoverer of the tetanus bacillus, while working under Koch.

The experiments conducted by Sir Charles Sherrington at the Lister Institute prove the specific value of the antitoxin beyond any doubt, and also give evidence of the enormous superiority of the intrathecal route of adminis- tration. The toxin was administered to monkeys, and after some time antitoxin was given, with the following results: twelve monkeys received intramuscular injections-all died ; eighteen monkeys received intrathecal injectionsonly five died. All the intrathecal injections were given through the atlanto-occipital membrane. (Both toxin and antitoxin were prepared at the Lister Institute.)

Sir David Bruce ${ }^{3}$ also notes these experiments, and considers the great value of the intrathecal administration of the antitoxin. Abercrombie ${ }^{4}$ finds that antitoxin is of decided value, the symptoms diminishing after its use, increasing after stoppage, and again diminishing when antitoxin is resumed. Such has been our experience also in a fair percentage of the cases. Stoppage of the antitoxin before all the symptoms had passed off was followed by exacerbations, which could immediately be brought under control by the administration of more serum. Ransom, ${ }^{5}$ in an extensive and well-reasoned review of tetanus, says that the therapeutic use of the antitoxin is fully justified, as all unbound toxin is neutralized, and therefore antitoxin at the right time may make all the difference in the outlook of a case.

With all the evidence and the opinions before us, there appears to be no doubt that the use of the antitoxin is quite rational and fully justified.

It is next necessary to consider why, in spite of the use of the antitoxin in large doses, the therapeutic results are not comparable with those obtained in diphtheria. Experimentally it has been proved that the antitoxin cannot combine with the toxin that has already reached the nerve cells. "The antitoxin cannot dislodge the toxin already combined with the nerve cell."' Ransom $^{5}$ states that the toxin in nerves and nerve cells is inaccessible to the antitoxin; the only toxin that can be neutralized is in the blood and lymph. This explains why antitoxin fails in a large proportion of cases-advanced ones with short incubation period-where the damage has already been done and the nerve cells are poisoned. It also means that the only hope of getting better results lies in early saturation of the blood with the antitoxin and the immediate neutralization of all toxin that lies free in the cerebro-spinal fluid.

\section{Administration and Results}

Antitoxin can be administered subcutaneously, intramuscularly, intravenously, and intrathecally-the last by a lumbar or cisterna magna puncture.

The best route has not yet been decided, and opinions differ regarding the relative value of the different routes. We have already seen that the experimental evidence on animals is strongly in favour of the intrathecal route. Sir William Leishman and Major Smallman, ${ }^{7}$ however, are not satisfied regarding the method of its administration, and although strongly advocating the use of the antitoxin, they say that the method of its administration leaves much to be desired. Ransom ${ }^{5}$ states that no toxin can be detected in the cerebro-spinal fluid experimentally, even after massive doses of the toxin ; although later he says, " antitoxin injected into the subarachnoid space would neutralize any toxin that might be there."

Permin $^{8}$ showed that if tetanus toxin was injected in the muscles in a suitable dose, local tetanus was prevented if antitoxin was given intrathecally at the same time, but was not prevented when the same was given intravenously. Golla ${ }^{9}$ also claims experimental superiority of the intrathecal and intravenous routes over the others. The memorandum on tetanus by the War Office Committee ${ }^{1}$ concludes that the intrathecal injections are the most effective.

Before I pass on to the consideration of the results obtained in this series of cases, it is necessary to say that no conclusions can be drawn from isolated cases or small groups of cases. We read in the medical literature 
of case reports, mostly of single cases, or short groups of six or ten, treated by certain methods, and later these methods are enthusiastically advocated. As in pneumonia, where fluctuations in the severity of the infection and in the mortality rates are notorious, so with tetanus-a series of cases may recover, although apparently serious, and with the same treatment another series of consecutive cases may die. Hence only figures from collections of large numbers of cases, spread out over a considerable number of years, are of value.

Two facts emerge, therefore, from the foregoing evidence.

1. Early neutralization of all circulating toxin by injections of large doses of the antitoxin, and the keeping up of the concentration of antitoxin by further and repeated intravenous and intramuscular injections, are necessary.

2. Intrathecal administration is justified, and is more useful than the other routes in the experimental infections.

N.B.-In most of the experimental work cited above the antitoxin was administered through the atlantooccipital membrane.

\section{Review of Cases}

In all I propose to review 278 cases. Of these I have taken 49 consecutive male cases admitted into the hospital in 1926 (before I took charge of the ward), which were being treated in a uniform manner (Group A). The records before these are not available. The other 229 cases have all been under my care. They are consecutive cases, and no selection whatever has been made. Fourteen of these had received no serum for some reason or another-a few were moribund, two very mild cases recovered, the rest died. The remainder are further subdivided into two main groups for purposes of comparison. Group B, 102 cases, was treated by varying doses of serum, the majority by intravenous and intramuscular routes, while twenty-two of them had intrathecal administration through a lumbar puncture in addition. The final 112 cases (Group $C)$, in addition to having the intramuscular and intravenous routes used, were all treated by intrathecal administration through the atlanto-occipital membrane into the cisterna magna.

\section{Group A}

The treatment followed to some extent as a routine before I took charge was somewhat as follows: bromides and chloral hydrate by mouth in moderate doses, a fair amount of morphine, and antitoxin in doses of about 20,000 units per day, intravenously and intramuscularly. Three cases had received 18,000 units intrathecally by the lumbar route.

Three patients were below the age of 10 , and five above the age of 40 . In twenty-six cases ( 53 per cent.) the incubation period could not be determined, in ten (20 per cent.) it wis eleven days or more, in three ( 7 per cent.) between six and ten days, and in ten (20 per cent.) between one and five days. There was no evidence or history of injury in twenty-four cases (49 per cent.), there were frankly septic wounds in twenty-two (45 per cent.), and nail injuries in three cases 6 per cent.). Treatment was similar in all cases with the exception of three, where serum had, in addition, been administered intrathecally by the lumbar route. Out of these forty-nine cases, eleven recovered and thirty-eight died, a mortality rate of $\mathbf{7 7 . 5}$ per cent. All the three in whom intrathecal administration had been made expired. Out of the thirty-eight fatal cases, seventeen died within twenty-four hours of admission. After excluding these from consideration, eleven out of thirty-two cases were cured and twenty-one expired-a mortality rate of 65.6 per cent.

\section{Group B}

When I took charge I started on the same lines, using somewhat larger doses of the serum and sedatives, and not much morphine, which in bad cases seemed to increase the tendency to pulmonary oedema, in spite of its being given with atropine. The average dose of the antitoxin was 30,000 to 40,000 units on the first day. The first eighty cases were treated in this way, with the result that twenty-eight of them recovered and fifty-two died (65 per cent.).
This was hardly satisfactory, and hence I thought of making additional use of the intrathecal route, by lumbar puncture, and further increasing the dose of the antitoxin. Twentytwo cases were thus treated, of whom eight (36 per cent.) recovered and fourteen (64 per cent.) died. The result, therefore, did not appear to be any more satisfactory than that in Group A. I admit, however, that this was a small number of cases, hence too much value cannot be attached to the figures. Clinically, in spite of two or three lumbar punctures and repeated administration of the antitoxin, the general effect was not much better, so that it was probable that the antitoxin did not diffuse high enough or quickly enough.

Taking both groups together we have 102 cases, with thirtyseven recoveries and sixty-five deaths-a mortality of over 64 per cent. In this group of cases the incubation period could not be determined in 45 per cent., was between one and five days in 19 per cent., between six and ten days in 20 per cent., and eleven days or above in 16 per cent. The percentages are quite comparable with the previous group of cases. There was no evidence of injury in 23 per cent., there were septic wounds in 62 per cent., and nail injuries in 15 per cent. In the previous group the percentage of septic and nail injuries was smaller. Thirty patients died within twenty-four hours; excluding these we have thirty-five deaths out of seventy-two cases-a mortality rate of 50 per cent.

\section{Technigue of Cisternal Puncture}

All experimental evidence was in favour of the utility of the intrathecal route for administration of the antitoxin, but still the results obtained by the lumbar route were hardly encouraging. As in all experimental work the injection was made through the atlanto-occipital membrane, one thought that the administration of the antitoxin in the cisterna magna through the atlanto-occipital space might prove more successful. Theoretically at least, the antitoxin would have a better chance of neutralizing the toxin that might be present near the vital centres. The technique of the cistern puncture is simple if ordinary precautions are taken. In over 140 punctures we have had no fatalities that could be directly attributed to the puncture. We have on six occasions not been able to get into the cisterna magna, owing either to its depth exceeding $6 \mathrm{~cm}$., or to the presence of a bony spur between the atlas and the occiput.

The cisterna magna is situated in front of the occipitoatlantoid ligament at a distance of 4.5 to $6 \mathrm{~cm}$. in the adult. Very rarely the distance may be greater, but for the sake of safety it is not advisable to go beyond $6 \mathrm{~cm}$. The hair at the back of the head is shaved. The tips of the mastoid processes are important landmarks. The centre of the line joining the tips of the mastoid processes is the point for puncture. It would be preferable to have the patient sitting up with the head flexed, but as this position is not possible in tetanus cases the patient is made to lie on one side with the head flexed as much as possible, this position being maintained by the assistants. Instantaneous anaesthesia may be obtained by the use of liq. somniferus. After flexing the head on the chest, tincture of iodine is applied, and if no anaesthetic has been given a little 1 per cent. novocain is infiltrated in the line of the puncture for 3 or $4 \mathrm{~cm}$. Even this has not been necessary in a large number of our cases. The lumbar puncture needle, which should preferably have a guard at $6 \mathrm{~cm}$., or a good mark at that distance, is then introduced in the centre of the line joining the tips of the mastoid processes and above the spine of the atlas, which is easily felt. The needle is pushed in slightly upwards till the edge of the foramen magnum is struck. The point of the needle is now slightly depressed and the atlanto-occipital ligament pierced. When the needle reaches the dura mater, and while it is piercing it, the patient feels a sharp twinge of pain. The needle should now be gently rotated, and the cerebro-spinal fluid then rushes out. It is wise to remove the trocar from time to time to see if the cistern is reached. The whole operation is done slowly and deliberately. As a rule the puncture is easv, and dry taps do not occur, as they sometimes do in lumbar puncture. Twenty to thirty c.cm. of the fluid is allowed to come out, and is then replaced by a slightly smaller amount of the antitoxin. This must be done very slowly, at least two to three minutes being taken. A syringe may be used, special care being taken not to force - the piston too quickly. It is preferable, however, to use the 
gravity method, the barrel of a 20-c.cm. syringe being connected by a small rubber tube to a metal piece which fits the needle, and the serum allowed to flow slowly in. This being done, the foot of the bed may be raised so as to keep the fluid at a high level for as long a time as possible. The neutralization of any free toxin that might be present in the cerebro-spinal fluid will be prompt.

\section{Group C}

One hundred and twelve cases have been treated thus, antitoxin having been administered intrathecally in the cisterna magna, as well as intravenously, intramuscularly, and subcutaneously-often around the wound, if one is present. The cases have been mostly consecutive, all the very severe ones being included. Out of these 112 cases, fifty-nine have recovered (53 per cent.), and fifty-three have died (46.5 per cent.). Considering the mortality of Group B (102 cases), which was 64 per cent., and of Group A (49 cases), which was 77 per cent. (before excluding cases in which death occurred within twenty-four hours), this last group of 112 cases shows an appreciable difference-15.5 per cent. as compared with Group B, and 30.5 per cent. as compared with Group A.

Though not spectacular, these figures are encouraging, and justify the intracisternal administration of the antitoxin.

In this group of 112 cases the incubation period could not be determined in forty-seven cases (42 per cent.), was between one and five days in twenty-four cases ( 21 per cent.), between six and ten days in twenty-five cases (22 per cent.), and eleven days and above in sixteen cases (15 per cent.). These figures are quite comparable with both the previous groups. There was no evidence of injury in 24 per cent., septic injuries in 66. per cent., and nail injuries in 10 per cent. The age of the vast majority of cases (74 per cent.) was between 11 and 40 , only 19 per cent. being above 40 , and 7 per cent., the remainder, below 10 . These figures are given as evidence that the groups of cases considered are comparable, as they are very nearly the same in age, incubation period, and type of injury. Of the fifty-three deaths in the last group, no fewer than thirty-five patients died within twenty-four hours, so that, excluding these cases, we have a total of seventy-seven cases out of which eighteen have died-a mortality rate of 23.4 per cent. This compares very favourably with the figures of 65.6 per cent. in Group A (small doses of antitoxin) and 50 per cent. in Group B (larger doses of antitoxin, but without administration into the cisterna magna).

\section{Discussion}

Without making any undue claims for these figures, and while admitting that the number of cases is not as large as one would wish, it appears that administration of antitoxin in the cisterna magna, in association with large doses intravenously, intramuscularly, and subcutaneously, is definitely indicated in all cases of tetanus, and, provided the patient has not been admitted in such a bad condition as to die within twenty-four hours, there is a 77 per cent. chance of his recovery. This is not so if small doses of serum only are administered, or even if larger doses are given without the intrathecal administration.

As a rule one puncture has sufficed in these cases, and in only half a dozen cases has more than one puncture been made. Clinically, in the majority of cases some improvement follows within twenty-four hours of the cistern puncture. The antitoxin appears to neutralize any toxin that may happen to be free in the cerebro-spinal fluid at the time of the injection. The patient is more comfortable, takes feeds more easily, and the lockjaw seems to improve more quickly. A certain amount of reaction is usual after the intrathecal administration, the most constant feature being a rise of temperature of about 2 to 3 degrees, and perhaps a slight increase in irritability. This usually subsides within twenty-four to forty-eight hours, and does not seem to do any harm. Serum reactions do occur, although no case of a severe anaphylactic reaction was seen in our series. The usual reactions have been erythematous rashes, joint pains with or without effusion, pyrexia, headache, etc. These have all yielded to adequate doses of calcium by mouth, although in a small number of cases intravenous injections of calcium chloride had to be given. Calcium was administered as a routine in all cases from the start as a preventive for serum reactions. The small number of cases in which the reaction developed is probably due to this.

The amount of antitoxin required varies considerably in each individual case, and no hard-and-fast rule can be laid down for it. Patients have recovered with 40,000 units, while others have required over 400,000 units. Each case has to be considered on its own merits, as procedure obviously depends on the severity of the primary infection, the incubation period, the site of injury, the vitality of the patient, and other factors. The administration should be kept up as long as there are spasms and general rigidity: this varies enormously in different cases. One often meets with cases where early diminution or stoppage of the serum has brought on fresh spasms or increased the rigidity, which has promptly subsided on the administration of more serum. Very large doses of the serum have been advocated on the Continent, in America, and elsewhere. Paterson ${ }^{10}$ of Australia advocates much larger doses-over 700,000 units per case-and claims better results. He reports only seven deaths in twenty-six consecutive cases.

As the value of convalescent serum has been established in some infective conditions, it may be profitable to try its use in tetanus. Serum might be collected from convalescent cases and preserved for use in the place of the usual antitoxin, though certain difficulties have first to be overcome. I feel that here we have an important problem. But though there is adequate clinical material the research facilities are somewhat defective.

\section{Conclusions}

It thus appears to be established that:

1. The use of tetanus antitoxin in the treatment of tetanus is perfectly rational, is absolutely necessary, and should be used early and in large doses.

2. The combined intrathecal (cistern puncture), intravenous, and intramuscular method of administration is superior to all the others. It definitely lowers the mortality rate, and ameliorates the symptoms far more than does treatment by intravenous and intramuscular routes alone.

3. The administration of the antitoxin into the cisterna magna is preferable to the lumbar puncture, as the former is nearer the vital centres. This method appears to neutralize any toxin that might be there quickly, and is much easier to perform.

4. The cistern puncture, properly performed, has no dangers. Not one single fatality could be attributed to the administration of the antitoxin by that route directly in a series of 112 cases. If the needle is not pushed beyond 5.5 to $6 \mathrm{~cm}$. in adults there is no danger of puncturing the medulla.

I cannot close this paper without thanking my successive house-physicians, without whose whole-hearted co-operation the treatment could not have been carried out so consistently and successfully. All of them had the opportunity of performing the cistern puncture. My thanks are due to the superintendent of the J. J. Hospital, Lieut.-Colonel S. S. Vazifdar, for putting at my disposal the large quantities of the serum required, even though the bill at the end of the month was sometimes staggering.

\section{ReFERENCES}

1 Memorandum on Tetanus by War Office Committee, British Medical Journal, 1916, ii, 647.

2 Howitt and Jones: Lancet, 1915, i, 753.

3 Hruce Sir David: Ibid., 1917, i, 680.

4 Aruce, Sir Daritish Medical Journal, 1916, i, 339.

5 Ransom: Lancet, 1917, ii, 928.

5 Ransom: Lancet,

' Ibid., 1917, i, 691.

${ }^{7}$ Leishman, Sir William, and

- Golla: Ibid., 1917, i, 686.

${ }^{10}$ Paterson: Med. Journ. of Australia, 1930, i, 832

${ }_{11}$ Pilloni: Policlinico-Rome, April 13th, 1931, p. 510.

12 Suvansu: Lancet, 1931, i, 1075. 